\title{
Neutrophilia Due to Silica Nanoparticles Induces Release of Double- Stranded DNA
}

\author{
Kazuma Higashisaka ${ }^{1,2}$, Akiyoshi Kunieda', Yuki Iwahara1, Kota Tanaka ${ }^{1}$, Kazuya Nagano², Yohei Mukai ${ }^{3}$, Haruhiko Kamada ${ }^{2,4}$, Shin-ichi \\ Tsunoda ${ }^{2,4}$, Yasuo Yoshioka ${ }^{1,2 *}$ and Yasuo Tsutsumi ${ }^{1,2 *}$ \\ ${ }^{1}$ Laboratory of Toxicology and Safety Science, Graduate School of Pharmaceutical Sciences, Osaka University, 1-6 Yamadaoka, Suita, Osaka 565-0871, Japan \\ ${ }^{2}$ Laboratory of Biopharmaceutical Research, National Institute of Biomedical Innovation, 7-6-8, Saito-Asagi, Ibaraki, Osaka 567-0085, Japan \\ ${ }^{3}$ Laboratory of Innovative Antibody Engineering and Design, Center for Drug Innovation and Screening, National Institute of Biomedical Innovation, 7-6-8 Saitoasagi, \\ Ibaraki, Osaka 567-0085, Japan \\ ${ }^{4}$ The Center for Advanced Medical Engineering and Informatics, Osaka University, 1-6, Yamadaoka, Suita, Osaka 565-0871, Japan
}

\begin{abstract}
Various types of nanomaterials have been developed for consumer and industrial applications, and the safety of such materials is the subject of considerable research around the world. Several studies have reported the inflammatory effects of nanomaterials, but the details of the involvement of neutrophils, the first leukocytes to be recruited to inflammation sites, in nanomaterial-induced inflammation are poorly understood. Here, we examined neutrophil activation in mice treated with silica particles. Twenty-four hours after treatment, the proportion of neutrophils in peripheral blood of mice injected with 70-nm-diameter silica nanoparticles (nSP70) was significantly higher than in saline-treated mice, whereas treatment with silica particles with diameters of 300 or $1000 \mathrm{~nm}$ did not result in any significant change in neutrophil proportion. In addition, higher plasma concentrations of myeloperoxidase were observed only in the nSP70-treated mice, and treatment with nSP70 surface-modified with amino groups did not elevate the proportion of neutrophils. Moreover, mice treated with antibodies to granulocyte colony-stimulating factor (G-CSF) exhibited a significant decrease in nSP70-induced neutrophilia relative to untreated mice, suggesting that nSP70induced neutrophilia resulted from G-CSF production induced by nSP70. In addition, we demonstrate that nSP70induced neutrophilia contributed to elevation of plasma concentrations of double-stranded DNA. Our results indicate that the nSP70-induced increase in the proportion of neutrophils depended on G-CSF elevation and that nSP70 may have induced the formation of neutrophil extracellular traps. Our results provide basic information about the association of neutrophil activation with silica nanoparticle-induced biological effects.
\end{abstract}

Keywords: Nanomaterial; Inflammation; Neutrophil; NETs

Abbreviations: CXCL2: Chemokine (C-X-C motif) Ligand 2; dsDNA: Double-Stranded DNA; G-CSF: Granulocyte ColonyStimulating Factor; nSP70: 70-nm Diameter Silica Nanoparticles; nSP70-N: nSP70 Surface-modified with Amino Groups; nSP300: 300nm Diameter Silica Particles; MPO: Myeloperoxidase; mSP1000: 1000nm Diameter Silica Particles; NETs: Neutrophil Extracellular Traps; PBS: Phosphate Buffered Saline

\section{Introduction}

Nanomaterials have been defined as materials with diameters of $\leq$ $100 \mathrm{~nm}$ that show properties different than those of the corresponding materials with diameters of $>100 \mathrm{~nm}$. They are expected to find numerous medical uses because of their many favorable properties [1]. Especially, owing to their surface characteristics and porosity, silica nanoparticles are expected to be useful for medical applications. For example, mesoporous silica nanoparticles have been designed for gene delivery [2], and amino-functionalized silica nanoparticles have the potential for use in vivo gene-therapy applications [3]. In addition, nanomaterials show enhanced permeation of and retention in tumor tissue relative to normal tissue, and they have been studied for use in drug-delivery systems, diagnostic and imaging systems, and various innovative therapeutic strategies $[4,5]$.

If nanomaterials are to be used for drug delivery, it is essential that both their quality and safety be established. However, many recent studies have shown that they may have unexpected biological effects that the corresponding conventional-sized materials do not [6,7]. For example, we have shown that compared to silica particles with diameters of $>100 \mathrm{~nm}$, silica nanoparticles with diameters of $\leq 100 \mathrm{~nm}$ are more likely to cause consumptive coagulopathy [8] and pregnancy complications in mice [9]. Moreover, several studies have reported that nanomaterials induce inflammatory responses, which occur when exogenous substances enter the body. However, the correlation of inflammatory responses with neutrophil activation is not yet well understood [10]. Neutrophils are known to be involved in various inflammatory responses; they are the first leukocytes to arrive at the site of invasion by an exogenous substance, and they have been shown to eliminate the causes of inflammation at the invasion site by means of various effects [11]. Pathogens are killed by reactive oxygen species produced by neutrophils, but inappropriate production of reactive oxygen species can aggravate various diseases and conditions involving inflammation, and excessive activation of neutrophils can injure tissues, damage DNA, and induce apoptosis in some chronic inflammatory conditions [12].

*Corresponding authors: Yasuo Yoshioka, Laboratory of Toxicology and Safety Science, Graduate School of Pharmaceutical Sciences, Osaka University 1-6, Yamadaoka, Suita, Osaka 565-0871, Japan, Tel: 81-6-6879-8230; Fax: 81-6-68798233; E-mail:yasuo@phs.osaka-u.ac.jp

Yasuo Tsutsumi, Laboratory of Toxicology and Safety Science, Graduate School of Pharmaceutical Sciences, Osaka University 1-6, Yamadaoka, Suita, Osaka 565-0871, Japan, Tel: 81-6-6879-8230; Fax: 81-6-6879-8233; E-mail: ytsutsumi@phs.osaka-u.ac.jp

Received September 09, 2014; Accepted October 14, 2014; Published October 23, 2014

Citation: Higashisaka K, Kunieda A, Iwahara Y, Tanaka K, Nagano K, et al. (2014) Neutrophilia Due to Silica Nanoparticles Induces Release of Double-Stranded DNA. J Nanomed Nanotechnol 5: 236. doi: 10.4172/2157-7439.1000236

Copyright: (c) 2014 Higashisaka K, et al. This is an open-access article distributed under the terms of the Creative Commons Attribution License, which permits unrestricted use, distribution, and reproduction in any medium, provided the original author and source are credited. 
Recent reports indicate that neutrophils may contribute to the elimination or degradation of nanomaterials. For example, it has been reported that single-walled carbon nanotubes are catalytically biodegraded in vitro by hypochlorite and reactive radical intermediates produced by the human neutrophil enzyme myeloperoxidase $[13,14]$ and that granulocytes may contribute to particle uptake in blood [15]. These results suggest that variation in the number of neutrophils recruited in response to invasion by nanomaterials may be associated with the in vivo kinetics of nanomaterials or with expression of the biological responses induced by nanomaterials. Thus, information about the relationship between nanomaterials and neutrophil activation can be expected to clarify not only the mechanism of unexpected biological effects of nanomaterials but the in vivo kinetics of nanomaterials. Here, we examined neutrophil activation induced by silica particles and tried to determine the association of neutrophil activation with silica nanoparticle-induced biological effects.

\section{Materials and Methods}

\section{Animals}

Female BALB/c mice (16 to $20 \mathrm{~g}$ ) were purchased from Nippon SLC (Shizuoka, Japan) and used in experiments at 6-8 weeks of age. The mice were housed in a ventilated animal room maintained at $20 \pm 2^{\circ} \mathrm{C}$ with a 12-h-light/12-h-dark cycle and free access to water and forage (FR-2, Funabashi Farm, Chiba, Japan). All of the animal experiments were performed in accordance with the Osaka University and National Institute of Biomedical Innovation guidelines for the welfare of animals.

\section{Materials}

Silica particles were purchased from Micromod Partikeltechnologie (Rostock/Warnemünde, Germany). Silica particles with diameters of 70,300 , and $1000 \mathrm{~nm}$ (nSP70, nSP300, and mSP1000, respectively) and nSP70 surface-modified with $\mathrm{NH}_{2}$ functional groups (nSP70-N) were used in this study. Prior to use, the particles were sonicated for $5 \mathrm{~min}$ and vortexed for $1 \mathrm{~min}$.

\section{Experimental protocols}

$\mathrm{BALB} / \mathrm{c}$ mice ( $n=5$ or 6 per group) were injected intravenously with $\mathrm{nSP} 70, \mathrm{nSP} 300, \mathrm{mSP} 1000$, or nSP70-N at $0.8 \mathrm{mg} / \mathrm{mouse}$ (about $40 \mathrm{mg} /$ $\mathrm{kg}$ ) or with saline. Blood samples were collected $24 \mathrm{~h}$ after treatment. For assessment of the effects of nSP70 dose, BALB/c mice ( $n=5$ or 6 per group) were injected intravenously with nSP70 at $0.2,0.4$, or $0.8 \mathrm{mg} /$ mouse (about 10, 20, or $40 \mathrm{mg} / \mathrm{kg}$ ). For evaluation of the time course of the response to treatment with the silica particles, $\mathrm{BALB} / \mathrm{c}$ mice $(n=5$ or 6 per group) were injected intravenously nSP70, nSP300, or mSP1000 at $0.8 \mathrm{mg} /$ mouse or with saline, and blood samples were collected 2 and 72 $\mathrm{h}$ after treatment. Plasma samples were obtained by centrifuging blood at $13,800 \times \mathrm{g}$ for $15 \mathrm{~min}$.

\section{Flow cytometry analysis}

Red blood cells in the collected blood samples were lysed with ammonium chloride. All staining procedures were performed in phosphate-buffered saline (PBS) containing $2 \%$ fetal calf serum. To minimize nonspecific binding, we preincubated single-cell suspensions with anti-CD16/CD32 antibodies (clone 93; eBioscience, San Diego, CA). Cells were labeled with combinations of phycoerythrin-conjugated Gr-1 antibodies (clone RB6-8C5; eBioscience), allophycocyaninconjugated CD11b antibodies (clone M1/70; BD Pharmingen, San Diego, CA), fluorescein isothiocyanate-conjugated F4/80 antibodies (clone CI:A3-1; AbD Serotec, Oxford, UK), and phycoerythrin-Cy7conjugated CD11c antibodies (clone HL3; BD Pharmingen). The cells were resuspended in staining buffer containing 7-amino-actinomycin $\mathrm{D}$ (BD Pharmingen), and the stained cells were analyzed for surface phenotype by means of a FACS Canto flow cytometer (BD Biosciences). The cells were gated according to side-scattered light (SSC) area and forward-scattered light (FSC) area, and the gated cells were then gated according to SSC height/SSC width, FSC height/FSC width, and 7-amino-actinomycin D to eliminate doublet cells and dead cells (parent population). Then, the proportion of neutrophils was calculated as the ratio of the neutrophil population $\left(\mathrm{CD} 11 \mathrm{~b}^{+} \mathrm{Gr}-1^{+} \mathrm{F} 4 / 80^{-}\right)$to the parent population.

\section{Hematology analysis}

Whole-blood samples were analyzed with a VetScan HMII Hematology System (Abaxis, Sunnyvale, CA) to determine white blood cell counts.

\section{Immunohistochemistry}

Approximately 20- $\mu \mathrm{m}$-thick frozen liver sections were fixed in cold acetone for $8 \mathrm{~min}$ and then blocked with 20\% fetal bovine serum in PBS for $1 \mathrm{~h}$. The blocked tissue sections were stained with LEAF Purified anti-mouse Ly-6G antibodies (1:500, BioLegend, San Diego, CA) or isotype controls (1:500, clone RTK2758; BioLegend) in PBS containing $12 \%$ bovine serum albumin (Sigma-Aldrich, St Louis, MO). The sections were washed in PBS and stained with secondary antibodies (Alexa Fluor 594 goat anti-rat IgG antibodies, 1:250, Invitrogen, Carlsbad, CA) in PBS containing $14 \%$ bovine serum albumin at room temperature for $1 \mathrm{~h}$. Finally, the sections were washed in PBS and mounted using VECTASHIELD mounting medium with 4',6-diamidino-2phenylindole (DAPI) (Vector Laboratories, Burlingame, CA). Images were visualized with an Olympus IX81 fluorescence microscope (Tokyo, Japan).

\section{Measurement of plasma concentrations of proteins and double-stranded DNA}

The plasma concentrations of myeloperoxidase (MPO) (Hycult Biotech, Plymouth Meeting, PA), granulocyte colony-stimulating factor (G-CSF) (R\&D Systems, Minneapolis, MN), and chemokine (C-X-C motif) ligand 2 (CXCL2) (R\&D Systems) were measured with a commercial enzyme-linked immunosorbent assay kit (ELISA). Extracellular double-stranded DNA (dsDNA) was quantified with a Quant-iT PicoGreen dsDNA Assay Kit (Invitrogen). All of the analyses were performed in accordance with the manufacturers' instructions.

\section{Quantification of G-CSF and CXCL2 concentrations in liver tissue supernatants}

$\mathrm{BALB} / \mathrm{c}$ mice ( $n=5$ or 6 per group) were injected intravenously with nSP70, nSP300, or mSP1000 (at $0.8 \mathrm{mg} /$ mouse) or with saline. Twenty-four hours after treatment, the animals were euthanized, and the livers were removed and stored at $-80^{\circ} \mathrm{C}$ prior to analysis. Each liver was homogenized with tissue lysis buffer consisting of $7 \mathrm{M}$ urea, $2 \mathrm{M}$ thiourea, 4\% [3-(3-cholamidepropyl) dimethylammonio1 -propanesulphonate], and $100 \times$ protease and phosphatase inhibitor cocktail (Thermo Scientific, Waltham, MA). The homogenates were centrifuged at $21,500 \times \mathrm{g}$ for $15 \mathrm{~min}$, and the supernatants were collected. ELISA for G-CSF and CXCL2 in the liver tissue supernatants were performed according to the manufacturer's protocol.

\section{In vivo neutrophil- and G-CSF-depletion protocols}

Neutrophil depletion was achieved by intraperitoneal injection of 50,100 , or $150 \mu \mathrm{g} /$ mouse anti-Ly-6G antibodies (clone $1 \mathrm{~A} 8$ 


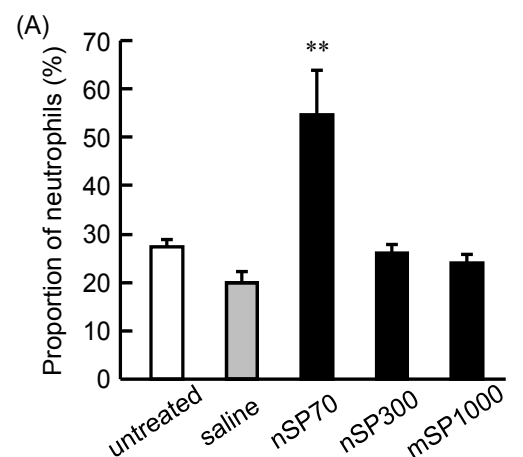

(B)

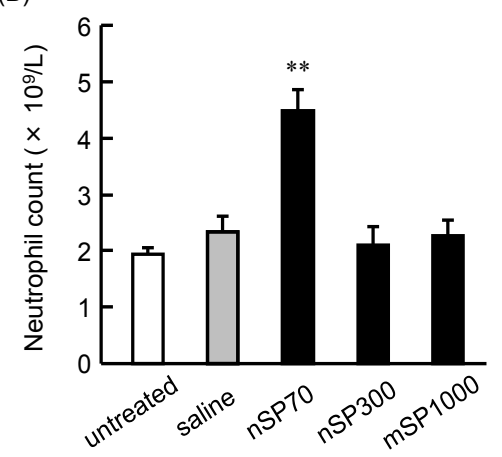

(C)

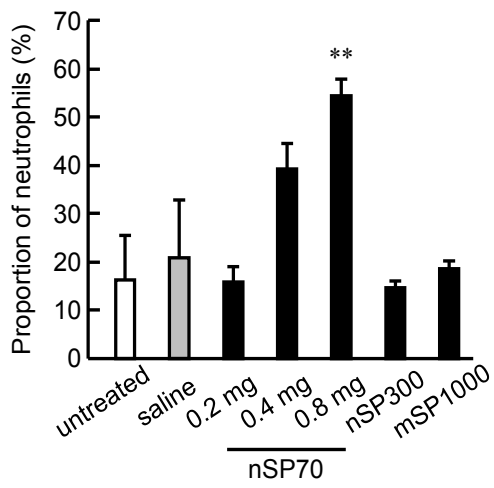

(D)

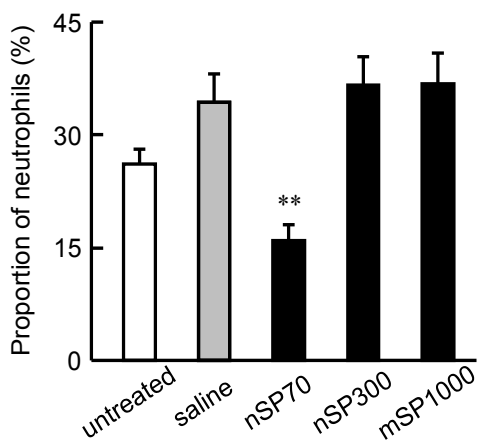

(E)

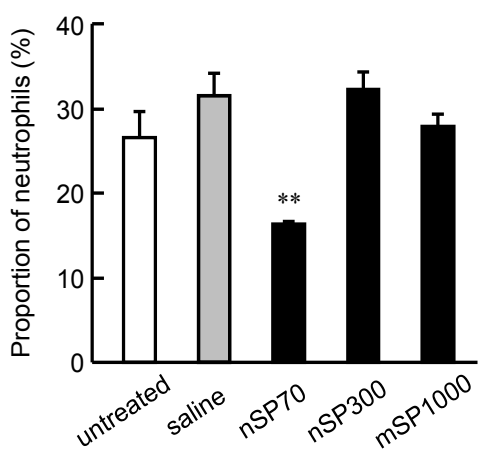

Figure 1: Effects of treatment with silica particles on neutrophil levels in mice. BALB/c mice were intravenously injected with nSP70 (0.8 mg/mouse), nSP300 (0.8 mg/mouse), mSP1000 (0.8 mg/mouse), or saline. BALB/c mice with no treatment were divided into untreated group. (A) The proportion of neutrophils in peripheral blood of each mouse was determined by flow cytometry $24 \mathrm{~h}$ after treatment. (B) The neutrophil count in peripheral blood of each mouse was calculated by multiplying neutrophil proportion by white blood cell count. (C) The effect of nSP70 dose $(0.2,0.4$, or $0.8 \mathrm{mg} / \mathrm{mouse})$ on the proportion of neutrophils in peripheral blood of each mouse was determined by flow cytometry $24 \mathrm{~h}$ after treatment. $(D, E)$ The proportion of neutrophils in peripheral blood of each mouse was determined by flow cytometry $2 \mathrm{~h}(\mathrm{D})$ and $72 \mathrm{~h}(\mathrm{E})$ after treatment. Data are presented as mean $\pm \mathrm{SEM} ; n=5-6$; ${ }^{* *} P<0.01$ vs saline-treated group.

BioLegend), isotype controls (clone RTK2758; BioLegend), or PBS into BALB/c mice ( $n=5$ or 6 per group) $24 \mathrm{~h}$ prior to nSP70 injection. G-CSF depletion was achieved by intraperitoneal injection of $50 \mu \mathrm{g} /$ mouse anti-G-CSF antibodies (clone 67604; R\&D Systems), isotype controls (clone 43414; R\&D Systems), or PBS into BALB/c mice $(n=5$ or 6 per group) $4 \mathrm{~h}$ prior to nSP70 injection.

\section{Statistical analyses}

All results are expressed as means \pm SEM. Differences were compared by means of Bonferroni's method by using Excel statistics software 2008 (SSRI, Tokyo).

\section{Results}

\section{Physical properties of silica particles}

Silica nanoparticles, which are among the most commonly used nanomaterials $[16,17]$, not only are used widely as cosmetics and food additives but are expected to find medical applications, particularly as gene-delivery vehicles. In this study, we used silica particles with diameters of $70 \mathrm{~nm}$ (nSP70), $300 \mathrm{~nm}$ (nSP300), and $1000 \mathrm{~nm}$ (mSP1000), the physical properties of which we assessed in a previous study [9]. Transmission electron microscopy analysis revealed that all the particles were smooth-surfaced spheres, and dynamic light 
Citation: Higashisaka K, Kunieda A, Iwahara Y, Tanaka K, Nagano K, et al. (2014) Neutrophilia Due to Silica Nanoparticles Induces Release of Double-Stranded DNA. J Nanomed Nanotechnol 5: 236. doi: 10.4172/2157-7439.1000236

Page 4 of 9

scattering measurements showed that the mean secondary particle sizes were $64.6,322$, and $1140 \mathrm{~nm}$ for $\mathrm{nSP} 70, \mathrm{nSP} 300$, and $\mathrm{mSP} 1000$, respectively.

\section{Analysis of proportion of neutrophils and neutrophil activation in mice treated with silica particles}

First, we intravenously injected mice with nSP70, nSP300, or $\mathrm{mSP} 1000$ and measured the proportion of neutrophils in peripheral blood by means of flow cytometry $24 \mathrm{~h}$ after treatment. Although the proportion of neutrophils in nSP300- and mSP1000-treated mice was not significantly affected by treatment, the proportion in mice treated with nSP70 at $0.8 \mathrm{mg} /$ mouse was significantly higher than the proportion in saline-treated mice (Figure 1A). We also calculated neutrophil counts by multiplying the proportion of neutrophils by the white blood cell count. The neutrophil count in nSP70-treated mice was significantly higher than the count in saline-treated mice (Figure 1B), suggesting that nSP70 induced neutrophilia in peripheral blood. We also found that the degree of nSP70-induced neutrophilia was dose dependent, increasing with increasing nSP70 dose (Figure 1C). In addition, we investigated the time course of the change in the proportion of neutrophils after treatment with the silica particles. At $2 \mathrm{~h}$ (Figure 1D) and $72 \mathrm{~h}$ (Figure 1E) after treatment, we observed no changes in the proportion of neutrophils in mice treated with nSP300 or mSP1000 compared to the proportion in saline-treated mice. However, in mice treated with nSP70, the proportions of neutrophils at both 2 and $72 \mathrm{~h}$ after treatment were significantly lower than the proportions in saline-treated mice at the same time points.

Next, we assessed neutrophil accumulation in the livers of mice $24 \mathrm{~h}$ after treatment with silica particles, because we previously reported that nSP70 might accumulated in the liver and induced severe liver damage [18]. Immunohistochemical analysis showed that the distribution patterns of neutrophils in the livers of nSP300-, mSP1000-, and saline-treated mice were almost equal (Figure 2). In contrast, more neutrophils were detected in the livers of nSP70-treated mice than in those of the saline-treated mice $24 \mathrm{~h}$ after treatment (Figure 2). These results indicate that nSP70 also induced neutrophilia in the liver.

MPO is one of the principal enzymes released from the azurophilic granules of activated neutrophils [19]. To determine whether nSP70 induced neutrophil activation, we determined the plasma concentrations of MPO in mice treated with silica particles. Comparison between mice treated with silica particles and saline-treated mice revealed that the plasma concentrations of MPO were higher in the nSP70-treated mice, but not in the nSP300- or mSP1000-treated mice (Figure 3A). Depletion of neutrophils by anti-Ly-6G antibodies, which are specific to neutrophils, partially suppressed the nSP70-induced MPO production (Figure 3B), suggesting that nSP70 activated neutrophils as well as increasing the proportion of neutrophils.

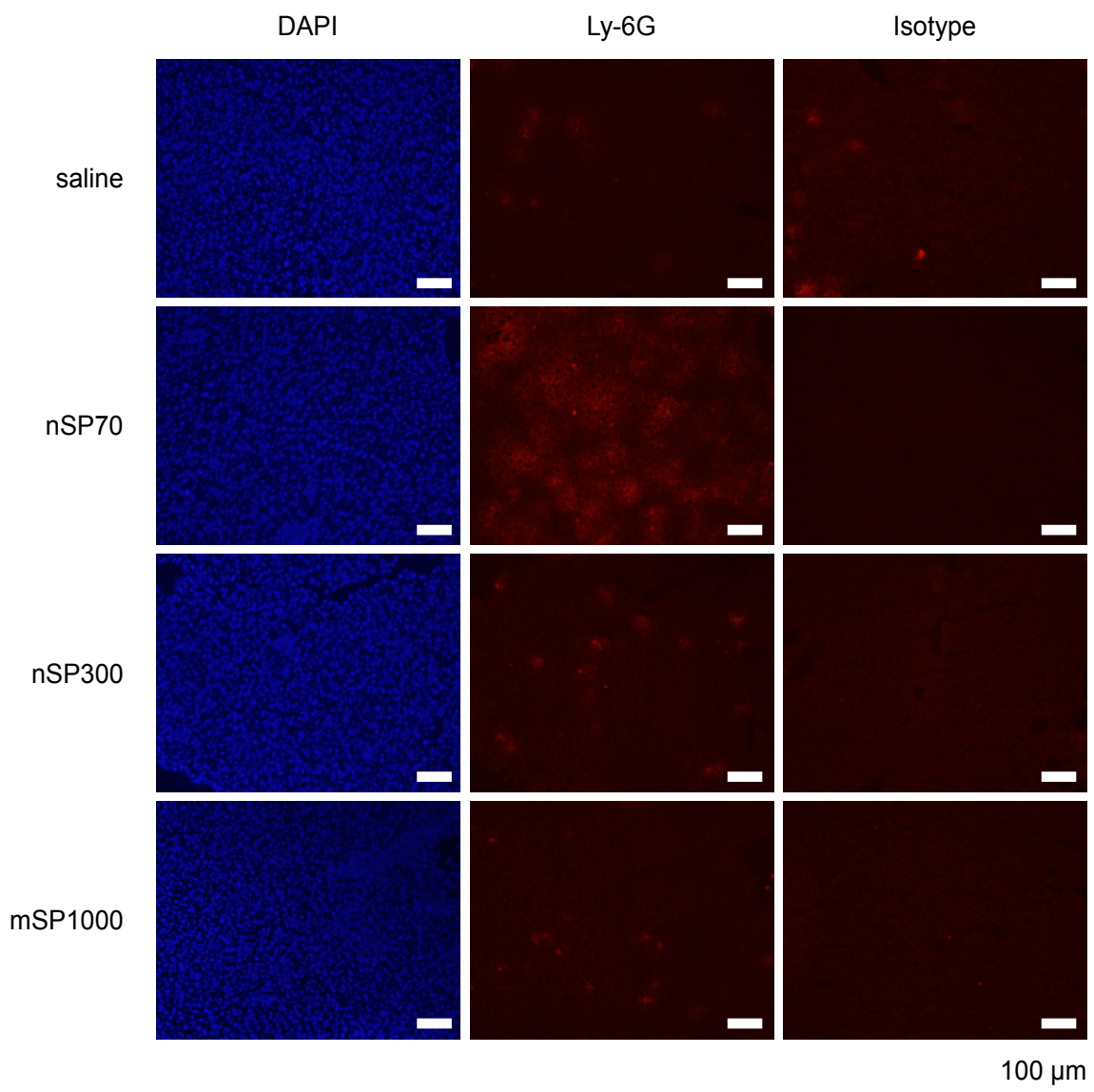

Figure 2: Fluorescence microscopy images showing neutrophil accumulation in mouse livers after treatment with silica nanoparticles. BALB/c mice $(n=5$ or 6 per group) were injected intravenously with nSP70, nSP300, or mSP1000 at $0.8 \mathrm{mg} / \mathrm{mouse}$ or with saline. After $24 \mathrm{~h}$, liver samples were collected, and frozen sections were prepared. The sections were stained with DAPI, anti-mouse Ly-6G antibodies, or isotype controls and were visualized with an Olympus IX81 fluorescence microscope. 
(A)

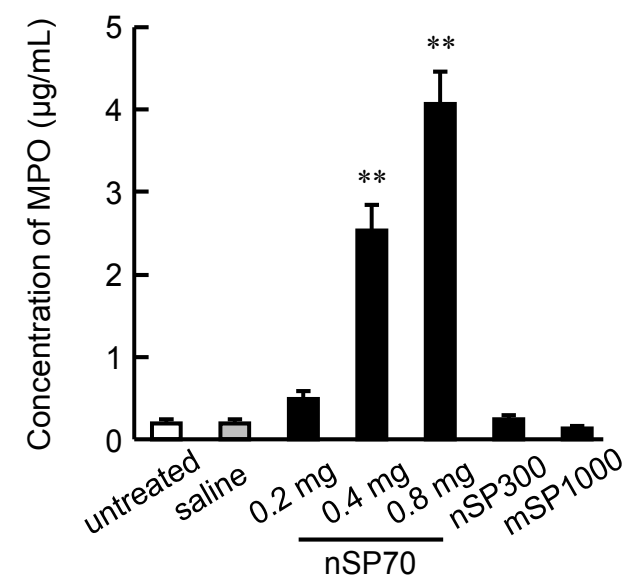

(B)

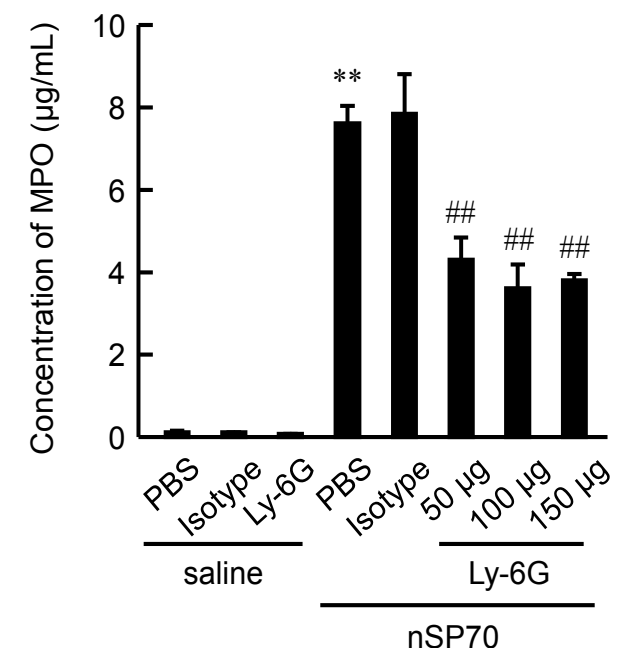

Figure 3: Effects of treatment with silica particles on plasma MPO levels. (A) $\mathrm{BALB} / \mathrm{c}$ mice were intravenously injected with $\mathrm{nSP} 70(0.2,0.4$, or $0.8 \mathrm{mg} / \mathrm{mouse})$, nSP300 (0.8 mg/mouse), mSP1000 (0.8 mg/mouse), or saline. BALB/c mice with no treatment were divided into untreated group. Plasma concentrations of MPO were determined by ELISA. (B) BALB/c mice were injected intraperitoneally with anti-Ly-6G antibodies (50,100, or $150 \mu \mathrm{g} / \mathrm{mouse}$ ), isotype controls (150 $\mu \mathrm{g} / \mathrm{mouse})$, or PBS $24 \mathrm{~h}$ prior to $\mathrm{nSP} 70$ injection $(0.8 \mathrm{mg} / \mathrm{mouse})$. Twenty-four hours after nSP70 injection, plasma concentrations of MPO were determined by ELISA. Data are presented as mean \pm SEM; $n=5-6 ;{ }^{* *} P<0.01$ vs saline-treated group; ${ }^{\#} P<0.01$ vs nSP70-treated group.

\section{G-CSF expression in mice treated with silica particles}

Next, to clarify the mechanism of nSP70-induced neutrophilia, we analyzed the expression of G-CSF, which plays essential roles in the proliferation and differentiation of neutrophils [20], in mice treated with each of the silica particles. The plasma concentrations of G-CSF in the nSP300- and mSP1000-treated mice $24 \mathrm{~h}$ after treatment were not significantly different from the concentration in saline-treated mice, whereas in nSP70-treated mice, the G-CSF concentration was significantly higher than that in saline-treated mice (Figure $4 \mathrm{~A}$ ). In addition, the G-CSF concentration in the liver of nSP70-treated mice $24 \mathrm{~h}$ after treatment was significantly higher than that in saline-treated mice (Figure 4B).

To investigate the relationship between nSP70-induced neutrophilia and the elevation of plasma G-CSF concentrations in nSP70-treated mice, we conducted G-CSF-depletion experiments. BALB/c mice were intraperitoneally injected with anti-G-CSF antibodies or isotype controls $4 \mathrm{~h}$ prior to $\mathrm{nSP} 70$ injection, and the proportion of neutrophils was analyzed $24 \mathrm{~h}$ after treatment with nSP70 (Figure 4C). The results showed that pretreatment with anti-G-CSF antibodies significantly suppressed the nSP70-induced elevation in the proportion of

(A)

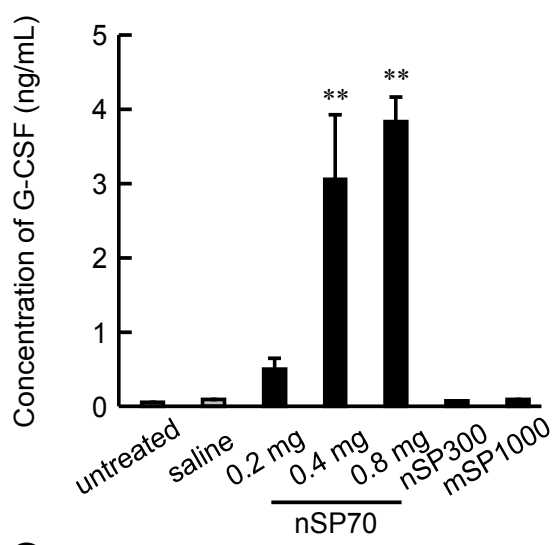

(B)

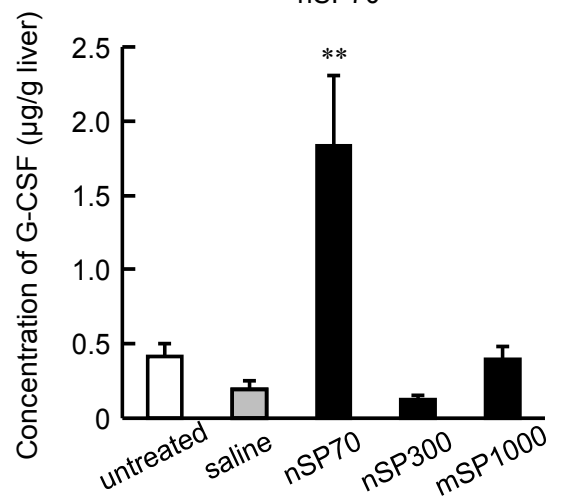

(C)

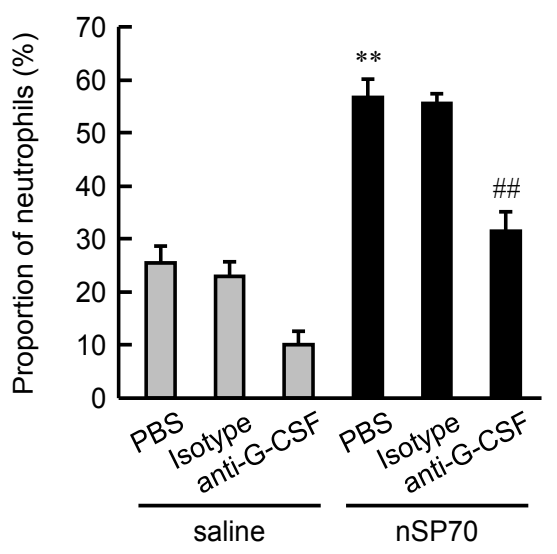

Figure 4: Effects of treatment with silica particles on G-CSF expression in mice. BALB/c mice were intravenously injected with $\mathrm{nSP} 70(0.2,0.4$, or $0.8 \mathrm{mg} /$ mouse), nSP300 (0.8 mg/mouse), mSP1000 (0.8 mg/mouse), or saline. BALB/c mice with no treatment were divided into untreated group. (A) The plasma concentrations of G-CSF were determined by ELISA $24 \mathrm{~h}$ after treatment. (B) The concentration of G-CSF in livers of mice was determined by ELISA 24 $\mathrm{h}$ after nSP70 injection (0.8 mg/mouse). (C) Anti-G-CSF antibodies $(50 \mu \mathrm{g} /$ mouse) or isotype controls were injected intraperitoneally into BALB/c mice $4 \mathrm{~h}$ prior to nSP70 injection ( $0.8 \mathrm{mg} / \mathrm{mouse})$. Twenty-four hours after nSP70 treatment, the proportion of neutrophils in peripheral blood of each mouse was analyzed by flow cytometry. Data are presented as mean \pm SEM; $n=5-6 ;{ }^{* *} P$ $<0.01$ vs saline-treated group; ${ }^{\star} P<0.05$ vs saline-treated group; ${ }^{\#} P<0.01$ vs nSP70-treated group. 
(A)

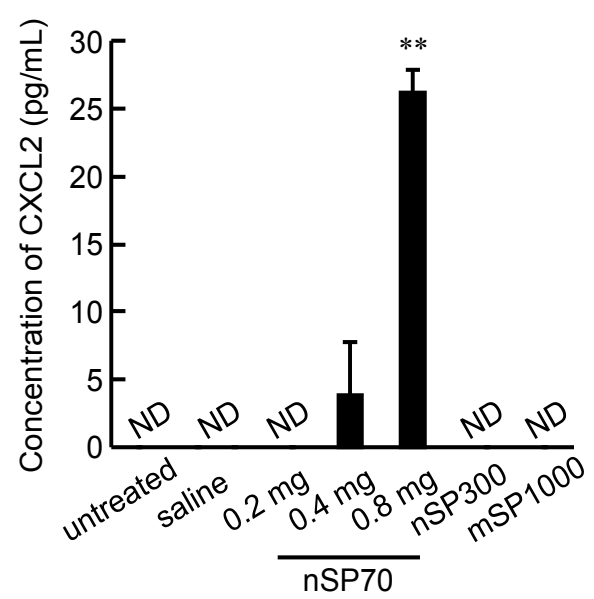

(B)

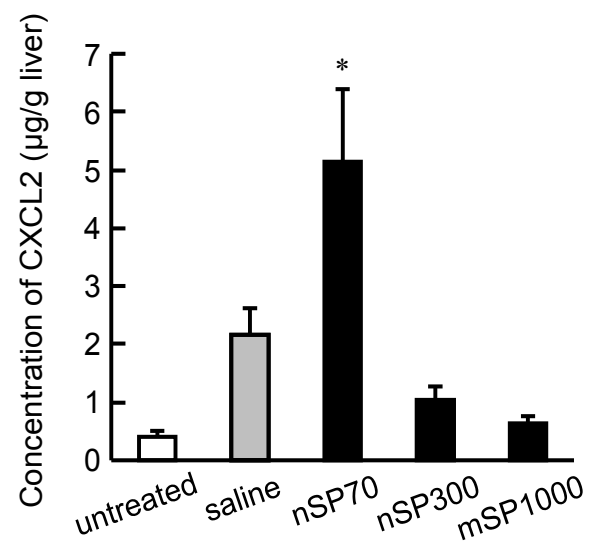

Figure 5: CXCL2 expression in mice treated with silica particles. BALB/c mice were injected intravenously with $\mathrm{nSP} 70(0.2,0.4$, or $0.8 \mathrm{mg} / \mathrm{mouse}), \mathrm{nSP} 300$ (0.8 mg/mouse), mSP1000 (0.8 mg/mouse), or saline. BALB/c mice with no treatment were divided into untreated group. (A) The plasma concentrations of CXCL2 were determined by ELISA $24 \mathrm{~h}$ after treatment. (B) The concentration of CXCL2 in livers of mice was determined by ELISA $24 \mathrm{~h}$ after nSP70 injection (0.8 $\mathrm{mg} /$ mouse). Data are presented as mean \pm SEM; $n=5-6$; ${ }^{* *} P<0.01$ vs salinetreated group; ${ }^{*} P<0.05$ vs saline-treated group; ND, not detected.

neutrophils. These results suggest that nSP70-induced neutrophilia was mediated by G-CSF production.

\section{CXCL2 expression in mice treated with silica particles}

Because recruitment of neutrophils to inflammation sites is mediated by CXCL2 (via the CXCR2 receptor on neutrophils) [21] and involves neutrophil migration from the bone marrow [22], we assessed the plasma concentrations of CXCL2 after treatment with silica nanoparticles. We found that neither nSP300 nor mSP1000 induced elevation of plasma concentrations of CXCL2, whereas treatment with nSP70 dose-dependently elevated plasma CXCL2 concentrations (Figure 5A). In addition, the concentration of CXCL2 in the livers of nSP70-treated mice was significantly higher than that in the livers of saline-treated mice (Figure 5B). These results suggest that nSP70induced neutrophilia resulted from nSP70-induced increases in the concentration of CXCL2.

\section{Responses of neutrophils to treatment with surface-modified nSP70}

The physical properties of nanomaterials, including surface properties and morphology, are important factors in biological responses to the materials $[23,24]$. Therefore, we evaluated how surface modification of nSP70 with amino groups affected the proportion of neutrophils in peripheral blood of treated mice. We previously reported that the mean secondary particle size of $\mathrm{nSP} 70-\mathrm{N}$ is $71.8 \mathrm{~nm}$, as indicated by dynamic light scattering measurements [9]. Unlike nSP70, nSP70-N did not induce increases in the proportion of neutrophils or the neutrophil count (Figures 6A and 6B). The plasma concentrations of both G-CSF (Figure 6C) and CXCL2 (Figure 6D) in nSP70-N-treated mice were significantly lower than those in nSP70-treated mice. These results suggest that surface-modified nSP70 induces inflammation to a lesser extent than unmodified nSP70.

\section{Quantification of dsDNA in mice treated with silica particles}

It has recently been reported that some activated neutrophils die and release neutrophil extracellular traps (NETs), structures composed of decondensed chromatin and antimicrobial proteins that trap a broad range of microbes and inhibit their dissemination [25,26]. We were interested in determining whether nSP70 would induce the formation of NETs. To quantify extracellular DNA, which is known to be a major component of NETs, we determined the plasma concentrations of dsDNA in mice treated with each of the silica particles. There were no significant differences in the concentrations of dsDNA in nSP300- and mSP1000-treated mice relative to the concentrations in the salinetreated mice (Figure 7A). However, the dsDNA concentrations in nSP70-treated mice were significantly higher than in saline-treated mice $24 \mathrm{~h}$ after treatment (Figure 7A). We also determined plasma dsDNA concentrations 2 and $72 \mathrm{~h}$ after treatment with silica nanoparticles and
(A)

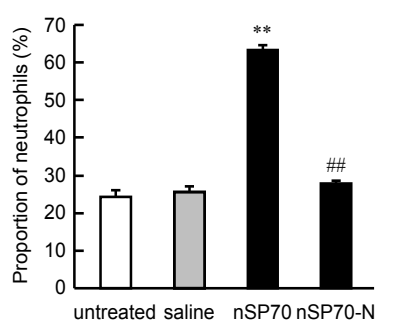

(B)

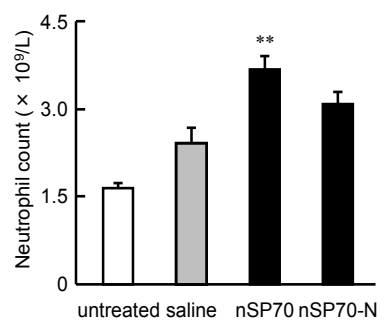

(C)

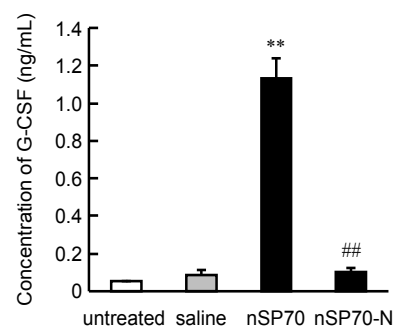

(D)

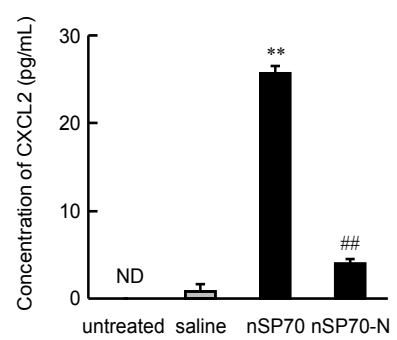

Figure 6: Effect of treatment with surface-modified nSP70 on neutrophil levels in mice. BALB/c mice were injected intravenously with nSP70 $(0.8 \mathrm{mg} / \mathrm{mouse})$ or nSP70-N (0.8 mg/mouse). BALB/c mice with no treatment were divided into untreated group. After $24 \mathrm{~h}$, (A) the proportion of neutrophils in peripheral blood of each mouse was determined by flow cytometry, and (B) the neutrophil count in peripheral blood of each mouse was calculated by multiplying neutrophil proportion by white blood cell count. Plasma concentrations of (C) G-CSF and (D) CXCL2 were determined by ELISA $24 \mathrm{~h}$ after treatment. Data are presented as mean \pm SEM; $n=5-6 ;{ }^{* *} P<0.01$ vs saline-treated group; ${ }^{\# \#} P<0.01$ vs nSP70-treated group; ND, not detected. 
(A)

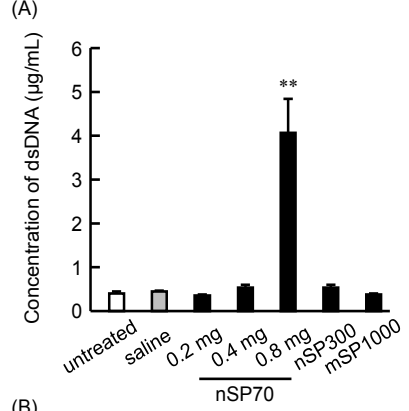

(B)

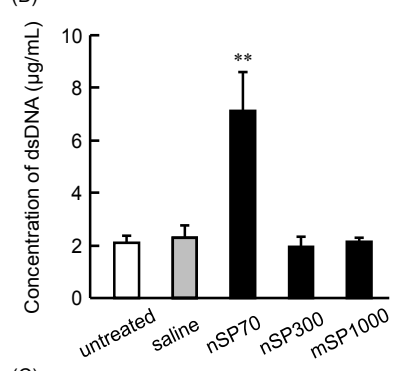

(C)

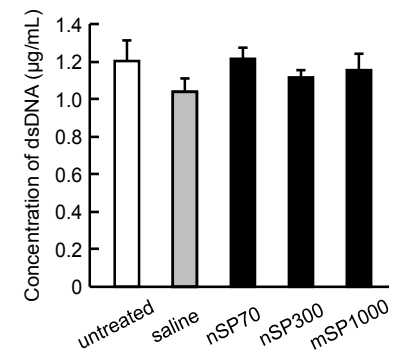

(D)

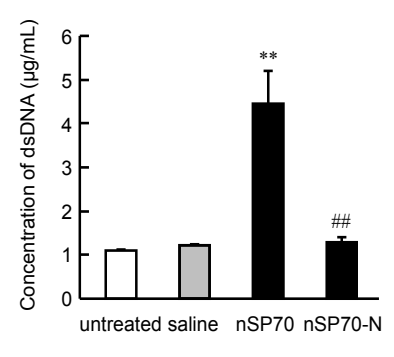

(E)

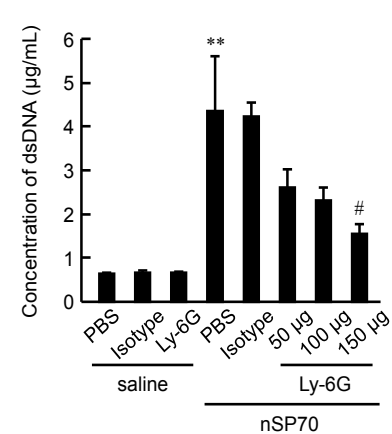

Figure 7: Plasma levels of dsDNA in mice treated with silica particles. BALB/c mice were injected intravenously with $\mathrm{nSP} 70(0.2,0.4$, or $0.8 \mathrm{mg} / \mathrm{mouse})$, nSP300 (0.8 mg/mouse), mSP1000 (0.8 mg/mouse), or saline. BALB/c mice with no treatment were divided into untreated group. (A) The plasma concentrations of dsDNA were determined at $24 \mathrm{~h}$ after treatment. $(B, C)$ The plasma concentration of dsDNA of each mouse was determined at $2 \mathrm{~h}$ (B) and $72 \mathrm{~h}(\mathrm{C})$ after treatment $(0.8 \mathrm{mg} / \mathrm{mouse})$. (D) BALB/c mice were injected intravenously with nSP70 (0.8 mg/mouse) or nSP70-N $(0.8 \mathrm{mg} / \mathrm{mouse})$, and the plasma concentration of dsDNA in each mouse was determined $24 \mathrm{~h}$ after treatment. (E) BALB/c mice were injected intraperitoneally with anti-Ly-6G antibodies $(50,100$, or $150 \mu \mathrm{g} /$ mouse), isotype controls $(150 \mu \mathrm{g} / \mathrm{mouse})$, or PBS $24 \mathrm{~h}$ prior to nSP70 injection $(0.8 \mathrm{mg} /$ mouse). The plasma concentration of dsDNA in each mouse was determined $24 \mathrm{~h}$ after nSP70 treatment. Data are presented as mean \pm SEM; $n=5-6$; ${ }^{*} P<0.01$ vs saline-treated group; ${ }^{\sharp} P<0.05$ vs nSP70-treated group.

found that nSP70 induced elevation of plasma dsDNA concentrations $2 \mathrm{~h}$ after treatment (Figure 7B) but that there was no significant difference in dsDNA concentrations between nSP70-treated mice and saline-treated mice $72 \mathrm{~h}$ after treatment (Figure $7 \mathrm{C}$ ). In contrast, treatment of mice with the surface-modified particles did not result in elevation of dsDNA concentrations compared to the concentrations in saline-treated mice (Figure 7D), and neutrophil depletion significantly suppressed dsDNA concentrations in nSP70-treated mice (Figure $7 \mathrm{E}$ ). These results suggest that nSP70-induced neutrophilia resulted in elevation of dsDNA concentrations and that nSP70 may induce the formation of NETs.

\section{Discussion}

Comparing the dose of the particles we used with the dose of particles used in other studies, some kinds of silica nanoparticles with surface modifications or functionalization have been used intravenously at a dose of $20 \mathrm{mg} / \mathrm{kg}$ to evaluate their therapeutic efficacy in mice $[27,28]$. On the other hand, some reports intraveneously administrated at several hundred milligrams per mouse of silica particles as drug delivery applications [27]. In this study, mice were intravenously injected at a dose of $0.8 \mathrm{mg} / \mathrm{mouse}$ (about $40 \mathrm{mg} / \mathrm{kg}$ ). Therefore, we consider that the dose in this study was typical of preclinical studies for drug delivery applications of silica particles.

It is not clear why only nSP70 induced an increase in the proportion of neutrophils. As particle size decreases, the particle unit of mass and overall specific surface area increases. According to the technical data sheets of the silica particles we used, nSP70, nSP300, and mSP1000 contain $2.8 \times 10^{12}, 3.5 \times 10^{10}$, and $9.5 \times 10^{8}$ particles $/ \mathrm{mg}$, respectively. That is, the specific surface areas of nSP70, nSP300, and mSP1000 are about $0.04,0.009$, and $0.002 \mathrm{~m}^{2} / \mathrm{mg}$, respectively. The larger specific surface area of nSP70 relative to nSP300 and mSP1000 may have allowed the smaller particles to interact with biomolecules such as proteins. In this study, we treated mice with equal masses of particles of different sizes. The specific surface area of $0.2 \mathrm{mg}$ of nSP70 (about $0.009 \mathrm{~m}^{2}$ ) is almost equal to that of $0.8 \mathrm{mg}$ of $\mathrm{nSP} 300$ (about $0.0072 \mathrm{~m}^{2}$ ), and the proportions of neutrophils in mice treated with the particles at these doses were equivalent (Figure 1C). Therefore, we suggest that the elevation in the proportion of neutrophils induced by silica particles might depend on the specific surface area of the particles. To confirm this possibility, it will be essential to examine the effects of silica particles by using doses with equal particle specific surface area concentrations.

We demonstrated that nSP70 induced neutrophilia (Figure 1A) by elevating the plasma concentration of G-CSF (Figure 4C). Recent studies have shown that serum amyloid A (SAA), an acute-phase protein, stimulates G-CSF expression in isolated macrophages and induces neutrophilia in mice [29]. We previously found that the plasma concentration of SAA is elevated in nSP70-treated mice but not in mice treated with nSP300 and mSP1000 [30] and that nSP70 were mainly detected in the liver [18]. Because the concentration of G-CSF in the liver was increased in nSP70-treated mice (Figure 4B) and because SAA is expressed by liver parenchymal cells $[31,32]$, it is conceivable that nSP70 might induce the release of SAA in the liver and the subsequent production of G-CSF from macrophages. We speculate that nSP70induced neutrophilia in the liver (Figure 2) occurred after SAA-induced G-CSF production in the liver. During inflammation, the number of neutrophils in tissues increases because neutrophils play a key role in eliminating pathogens and promoting tissue healing. Therefore, we suggest that nSP70-induced neutrophilia in the liver might contribute to elimination of $\mathrm{nSP} 70$.

In this study, we showed that the plasma concentrations of G-CSF (Figure 4A) and CXCL2 (Figure 5A) were elevated in nSP70-treated mice. The number of circulating neutrophils increases as a result of the rapid mobilization of neutrophils from bone marrow, and this mobilization is mediated by inflammatory mediators including CXCR2-binding chemokines such as CXCL1 and CXCL2 [22]. It has been demonstrated that G-CSF administration rapidly induces the production of CXCR2-binding chemokines, which in turn trigger enhanced motility of neutrophils in bone marrow and neutrophil mobilization into the circulation [21,33]. Therefore, we consider that nSP70-induced G-CSF elevation effected the production of CXCL2 and subsequent induction of neutrophilia.

Neutrophils are reported to be recruited to sites of infection or inflammatory stimuli within minutes [34]. We detected nSP70 in the liver of mice within $2 \mathrm{~h}$ of injection (unpublished data); therefore, it is 
conceivable that prompt recruitment of neutrophils to the liver might have resulted in the transient decrease in the proportion of neutrophils in peripheral blood observed $2 \mathrm{~h}$ after administration of nSP70 (Figure 1D). During the neutrophil life-span of a few days, essential processes involved in restoring homeostasis after nSP70-induced neutrophilia may result in the decrease in the proportion of neutrophils observed 72 $\mathrm{h}$ after nSP70 injection (Figure 1E).

Neutrophils recruited to sites of inflammation undergo an oxidative burst and form NETs, which are extracellular DNA fibers comprising histones and neutrophil-derived antimicrobial proteins [25]. NETs have been shown to have antimicrobial functions and to have proven benefit against infections [26,35]. Some studies have demonstrated that aggregation of NETs promotes the resolution of neutrophilic inflammation by degrading cytokines and chemokines [36]. Therefore, we speculate that nSP70-induced neutrophilia may protect against the biological effects, including inflammation, induced by nSP70.

nSP70 surface-modified with amino groups induced neutrophilia to a lesser extent than did unmodified nSP70 (Figure 6A), suggesting that modified nSP70 has little potential to induce inflammation. We previously demonstrated that surface-modified nSP70 is unlikely to induce undesired inflammatory responses in vitro and in vivo even though both unmodified and surface-modified nSP70 are equally taken up into macrophages [37,38]. Nanomaterials are reported to interact with proteins in such a way that a coating, known as the protein corona, forms around the nanomaterials [39]. Many studies suggest that the protein corona plays an important role in the biological effects and distribution of nanomaterials and that the proteinnanomaterial biological interaction depends on the surface properties of the nanomaterials [40]. Therefore, we suggest that the differences in protein binding due to differences in the physical properties of nanomaterials may induce different cellular responses, including inflammatory responses, and that the ability to control nanomaterial physical properties may lead not only to the clarification of the mechanism of action of nanomaterials but also to the development of safer nanomaterials.

\section{Acknowledgement}

This study was supported by Grants-in-Aid for Scientific Research from the Ministry of Education, Culture, Sports, Science and Technology of Japan (MEXT) and from the Japan Society for the Promotion of Science (JSPS); by Health Labour Sciences Research Grants from the Ministry of Health, Labour and Welfare of Japan (MHLW); by The Takeda Science Foundation; by The Research Foundation for Pharmaceutical Sciences; by The Japan Food Chemical Research Foundation; by Urakami Foundation; and by Uehara Memorial Foundation.

\section{References}

1. Wilczewska AZ, Niemirowicz K, Markiewicz KH, Car H (2012) Nanoparticles as drug delivery systems. Pharmacol Rep 64: 1020-1037.

2. Knopp D, Tang D, Niessner R (2009) Review: bioanalytical applications of biomolecule-functionalized nanometer-sized doped silica particles. Anal Chim Acta 647: 14-30

3. Bharali DJ, Klejbor I, Stachowiak EK, Dutta P, Roy I, et al. (2005) Organically modified silica nanoparticles: a nonviral vector for in vivo gene delivery and expression in the brain. Proc Natl Acad Sci U S A 102: 11539-11544.

4. Jain RK, Stylianopoulos T (2010) Delivering nanomedicine to solid tumors. Nat Rev Clin Oncol 7: 653-664.

5. Cheng Z, Al Zaki A, Hui JZ, Muzykantov VR, Tsourkas A (2012) Multifunctional nanoparticles: cost versus benefit of adding targeting and imaging capabilities. Science 338: 903-910

6. Elsaesser A, Howard CV (2012) Toxicology of nanoparticles. Adv Drug Deliv Rev 64: 129-137.

7. Arora S, Rajwade JM, Paknikar KM (2012) Nanotoxicology and in vitro studies: the need of the hour. Toxicol Appl Pharmacol 258: 151-165.
8. Nabeshi H, Yoshikawa T, Matsuyama K, Nakazato Y, Arimori A, et al. (2012) Amorphous nanosilicas induce consumptive coagulopathy after systemic exposure. Nanotechnology 23: 045101.

9. Yamashita K, Yoshioka Y, Higashisaka K, Mimura K, Morishita Y, et al. (2011) Silica and titanium dioxide nanoparticles cause pregnancy complications in mice. Nat Nanotechnol 6: 321-328.

10. Goncalves DM, de Liz R, Girard D (2011) Activation of neutrophils by nanoparticles. ScientificWorldJournal 11: 1877-1885.

11. Kolaczkowska E, Kubes $P$ (2013) Neutrophil recruitment and function in health and inflammation. Nat Rev Immunol 13: 159-175.

12. Eyles JL, Roberts AW, Metcalf D, Wicks IP (2006) Granulocyte colonystimulating factor and neutrophils--forgotten mediators of inflammatory disease. Nat Clin Pract Rheumatol 2: 500-510.

13. Kagan VE, Konduru NV, Feng W, Allen BL, Conroy J, et al. (2010) Carbon nanotubes degraded by neutrophil myeloperoxidase induce less pulmonary inflammation. Nat Nanotechnol 5: 354-359.

14. Farrera C, Bhattacharya K, Lazzaretto B, Andón FT, Hultenby K, et al. (2014) Extracellular entrapment and degradation of single-walled carbon nanotubes. Nanoscale 6: 6974-6983.

15. Jones SW, Roberts RA, Robbins GR, Perry JL, Kai MP, et al. (2013) Nanoparticle clearance is governed by Th1/Th2 immunity and strain background. J Clin Invest 123: 3061-3073.

16. Martin KR (2007) The chemistry of silica and its potential health benefits. J Nutr Health Aging 11: 94-97.

17. Popat A, Hartono SB, Stahr F, Liu J, Qiao SZ, et al. (2011) Mesoporous silica nanoparticles for bioadsorption, enzyme immobilisation, and delivery carriers. Nanoscale 3: 2801-2818.

18. Nabeshi H, Yoshikawa T, Matsuyama K, Nakazato Y, Matsuo K, et al. (2011) Systemic distribution, nuclear entry and cytotoxicity of amorphous nanosilica following topical application. Biomaterials 32: 2713-2724.

19. Lau D, Mollnau H, Eiserich JP, Freeman BA, Daiber A, et al. (2005) Myeloperoxidase mediates neutrophil activation by association with $\mathrm{CD} 11 \mathrm{~b} /$ CD18 integrins. Proc Natl Acad Sci U S A 102: 431-436.

20. Semerad CL, Liu F, Gregory AD, Stumpf K, Link DC (2002) G-CSF is an essential regulator of neutrophil trafficking from the bone marrow to the blood. Immunity 17: 413-423.

21. Köhler A, De Filippo K, Hasenberg M, van den Brandt C, Nye E, et al. (2011) G-CSF-mediated thrombopoietin release triggers neutrophil motility and mobilization from bone marrow via induction of Cxcr2 ligands. Blood 117: 43494357.

22. Burdon PC, Martin C, Rankin SM (2008) Migration across the sinusoidal endothelium regulates neutrophil mobilization in response to ELR + CXC chemokines. Br J Haematol 142: 100-108.

23. Nabeshi H, Yoshikawa T, Arimori A, Yoshida T, Tochigi S, et al. (2011) Effect of surface properties of silica nanoparticles on their cytotoxicity and cellular distribution in murine macrophages. Nanoscale Res Lett 6: 93.

24. Yoshida T, Yoshioka Y, Matsuyama K, Nakazato Y, Tochigi S, et al. (2012) Surface modification of amorphous nanosilica particles suppresses nanosilicainduced cytotoxicity, ROS generation, and DNA damage in various mammalian cells. Biochem Biophys Res Commun 427: 748-752.

25. Brinkmann V, Reichard U, Goosmann C, Fauler B, Uhlemann Y, et al. (2004) Neutrophil extracellular traps kill bacteria. Science 303: 1532-1535.

26. Jenne CN, Wong CH, Zemp FJ, McDonald B, Rahman MM, et al. (2013) Neutrophils recruited to sites of infection protect from virus challenge by releasing neutrophil extracellular traps. Cell Host Microbe 13: 169-180.

27. Li L, Tang F, Liu H, Liu T, Hao N, et al. (2010) In vivo delivery of silica nanorattle encapsulated docetaxel for liver cancer therapy with low toxicity and high efficacy. ACS Nano 4: 6874-6882.

28. Huang X, Li L, Liu T, Hao N, Liu H, et al. (2011) The shape effect of mesoporous silica nanoparticles on biodistribution, clearance, and biocompatibility in vivo. ACS Nano 5: 5390-5399.

29. He RL, Zhou J, Hanson CZ, Chen J, Cheng N, et al. (2009) Serum amyloid A induces G-CSF expression and neutrophilia via Toll-like receptor 2. Blood 113: 429-437. 
Citation: Higashisaka K, Kunieda A, Iwahara Y, Tanaka K, Nagano K, et al. (2014) Neutrophilia Due to Silica Nanoparticles Induces Release of Double-Stranded DNA. J Nanomed Nanotechnol 5: 236. doi: 10.4172/2157-7439.1000236

30. Higashisaka K, Yoshioka Y, Yamashita K, Morishita Y, Fujimura M, et al. (2011) Acute phase proteins as biomarkers for predicting the exposure and toxicity of nanomaterials. Biomaterials 32: 3-9.

31. Gabay C, Kushner I (1999) Acute-phase proteins and other systemic responses to inflammation. N Engl J Med 340: 448-454.

32. Yamamoto K, Shiroo M, Migita S (1986) Diverse gene expression for isotypes of murine serum amyloid A protein during acute phase reaction. Science 232 227-229.

33. Devi S, Wang Y, Chew WK, Lima R, A-González N, et al. (2013) Neutrophi mobilization via plerixafor-mediated $\mathrm{CXCR} 4$ inhibition arises from lung demargination and blockade of neutrophil homing to the bone marrow. J Exp Med 210: 2321-2336.

34. Fournier BM, Parkos CA (2012) The role of neutrophils during intestinal inflammation. Mucosal Immunol 5: 354-366.

35. Buchanan JT, Simpson AJ, Aziz RK, Liu GY, Kristian SA, et al. (2006) DNase expression allows the pathogen group A Streptococcus to escape killing in neutrophil extracellular traps. Curr Biol 16: 396-400.
36. Schauer C, Janko C, Munoz LE, Zhao Y, Kienhöfer D, et al. (2014) Aggregated neutrophil extracellular traps limit inflammation by degrading cytokines and chemokines. Nat Med 20: 511-517.

37. Morishige T, Yoshioka Y, Inakura H, Tanabe A, Yao X, et al. (2010) The effect of surface modification of amorphous silica particles on NLRP3 inflammasome mediated IL-1beta production, ROS production and endosomal rupture. Biomaterials 31: 6833-6842.

38. Morishige T, Yoshioka Y, Inakura H, Tanabe A, Narimatsu S, et al. (2012) Suppression of nanosilica particle-induced inflammation by surface modification of the particles. Arch Toxicol 86: 1297-1307.

39. Lundqvist M, Stigler J, Elia G, Lynch I, Cedervall T, et al. (2008) Nanoparticle size and surface properties determine the protein corona with possible implications for biological impacts. Proc Natl Acad Sci U S A 105: 14265-14270.

40. Monopoli MP, Aberg C, Salvati A, Dawson KA (2012) Biomolecular coronas provide the biological identity of nanosized materials. Nat Nanotechnol 7: 779786 